\title{
Seismic Analysis of the Transportation Portal by the Combined Asymptotic Method
}

\begin{abstract}
Alexander Tyapin ${ }^{1,2}$
${ }^{1}$ 1-119 Slaviansky Boulevard, Moscow 121352, Russia

${ }^{2}$ JSC Atomenergoproject, Moscow, Russia

Correspondence should be addressed to Alexander Tyapin, atyapin@bvcp.ru

Received 6 May 2011; Accepted 17 August 2011

Academic Editor: Leon Cizelj

Copyright ( 2011 Alexander Tyapin. This is an open access article distributed under the Creative Commons Attribution License, which permits unrestricted use, distribution, and reproduction in any medium, provided the original work is properly cited.

The author extends the previously proposed combined asymptotic method (CAM) of seismic SSI analysis for the multi-support systems and applies it to the transportation portal as a double-support system (together with the reactor building). The key issue is the development of the structural dynamic stiffness matrix condensed to the supports by the modal approach. Then the condensed structural matrix is combined with the soil dynamic stiffness matrix also condensed to the rigid basements. As a result, a very simple linear system $12 * 12$ is solved in the frequency domain. This gives the transfer functions from the free-field motion to the motion of the basements. The only important limitations are the linearity of the soil's and structure's properties and the rigidity of the basements. The results for the sample system are checked against the full SASSI solution. The results can be used to justify the further simplification of the system.
\end{abstract}

\section{Introduction}

Transportation portal (TP) is an important structure of a nuclear power plant (NPP). It is a frame resting on a separate basement. The frame is linked to the reactor building (RB) by horizontal beams (usually two pairs of them one under another). These beams have hinges in both ends. Thus, we have a double-support structure (TP + RB).

Seismic analysis of TP has some special features. First of all, horizontal beams support the railways for the crane (the upper pair) and the carriage (the lower pair). Both crane and carriage may be loaded or not; their positions on the ways may be different. Thus, one has to consider several variants of the system, different in masses and positions. It is not obvious what variant is more dangerous; different variants may be dangerous in different terms of response. So, one has to use a flexible tool to perform such an analysis.

Another special feature is a comparatively deep embedment of the portal basement. Hence, the surface model sometimes used for RB is not appropriate for the portal: the SSI must be treated in full.

In this paper the author extends the previously proposed combined asymptotic method (CAM) of seismic SSI analysis [1] for the multi-support systems and applies it to the transportation portal together with the reactor building as a double-support system. The key issue is the development of the structural dynamic stiffness matrix condensed to the supports. This issue was discussed in detail in [2].

The goal of this paper is to provide a CAM-based method to obtain the full solution considering the interaction of two basements both through the structure and through the soil foundation. The proposed method is applied to the comparatively simple model to verify the results against the benchmark solution obtained directly (i.e., without CAM). If the proposed method is correct, the results should be in good agreement with those obtained by direct analysis.

This full solution will be further used as a benchmark for the justification of the simplified approaches to this problem, neglecting one or another part of interaction (e.g., neglecting the back influence of portal to the RB motion or neglecting structure-soil-structure interaction).

\section{Generalization of CAM to Double-Support Systems}

The combined asymptotic method (CAM) is as follows. All basements are supposed to be rigid with 6 degrees of freedom (DOFs) each, thus making the number of DOFs 


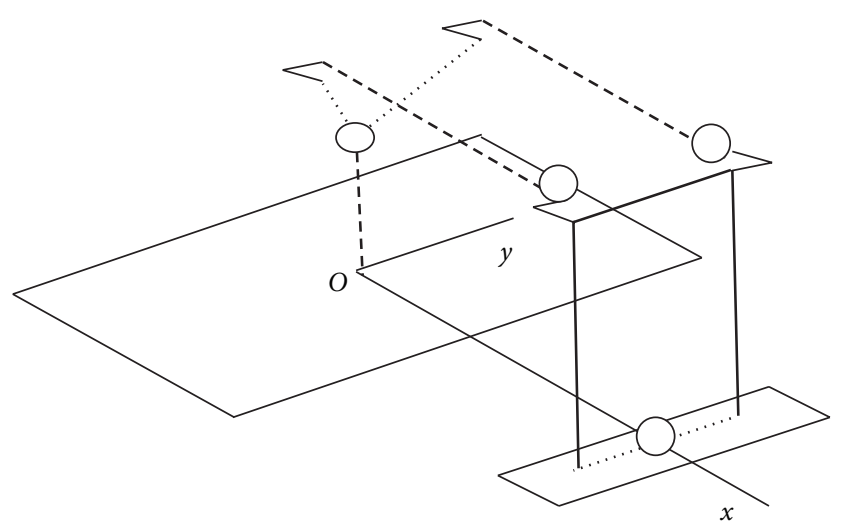

- Inertial flexible beams (portal frame)
-- Weightless flexible beams (RB and horizontal beams)
$\ldots$. Weightless rigid beams (portal basement and
links of the supports with RB)
Lumped masses having rotational inertia

FIGURE 1: Scheme of the sample structure modeling the reactor building (RB) and transportation portal (TP). Basements' contours are for the readability only.

for the double-support system $2 * 6=12$. The dynamic stiffness matrix of the structure is condensed to the supports and transforms into matrix $D$ of size $12 * 12$ (four blocks $6 * 6$ each; 12 for double-support system) - this is a complex frequency-dependent matrix [2]. In parallel, the dynamic stiffness matrix of the foundation is also condensed to the same rigid basements and transforms into matrix $S$ of the same size and block structure. Details of the soil stiffness condensation are given in [3]. Besides, seismic forces acting to the fixed basements due to the seismic wave in the foundation are also condensed to the basements into matrix $B$ of size $12 * 3$. This is a matrix of the transfer functions from the three-component free-field translational motion to the integral six component forces acting to each of two basements [3]. The unknown matrix $U$ of the transfer functions from the free-field motion to the motion of the basements has the same size $12 * 3$ as matrix $B$. It can be obtained from the equilibrium of the soil-structure contact surfaces treated as very thin weightless rigid shells. This equilibrium is described by a very simple linear system with complex coefficients:

$$
(D+S) U=B
$$

System (1) is of size 12 and is easily solved, giving the transfer functions matrix $U$ in the frequency domain. Time histories of the basements' motion are further obtained using fast Fourier transform (FFT) and time histories of the threecomponent free-field motion. After that one can perform standard multi-base time-domain calculations without soilstructure interaction (SSI).

One is usually interested also in the soil-structure interaction forces to check the capability of the soil foundation. These forces may be calculated after the solution of (1); this is done either from the soil or from the structure. The transfer functions from the free-field motion to the SSI forces are as follows:

$$
N=D U=B-S U .
$$

In fact (2) suggests two variants of the SSI forces calculation: through the structure $(D U)$ or through the soil $(B-S U)$. The result should be the same; the choice is by convenience of the execution.

Making a summary, ABAQUS is used here in the traditional mode. The difference with the single-based case CAM is that the modal analysis of the multi-supported structure is performed not once, but twice (once for all bases fixed, then for a single base fixed).

Unlike ABAQUS, SASSI is used in a somewhat unusual mode (condensed stiffness matrix and load matrix are obtained by special soft inserted in the SASSI [3]). However, the same results can be obtained using usual SASSI mode with MOTOR module [4].

\section{Application of CAM to the Seismic Analysis of Portal}

Let us consider the comparatively simple sample structure, reproducing the most important features of the real NPP portal. The structure is shown in Figure 1.

The main building (RB) is modeled by weightless vertical beam 24 meters high bearing the lumped mass on the top. This mass is equal to 250 thousand tones. The rotational inertia is $1.6 \mathrm{E} 7 \mathrm{t} \mathrm{m}^{2}$ around vertical axis $\mathrm{Oz}$ and around the longer axis $O y ; 1.9 \mathrm{E} 7 \mathrm{t} \mathrm{m}^{2}$ around the shorter axis $O x$; these are more or less practical values. Vertical beam has a circular cross-section with radius 16 meters; this radius provides the first natural frequency of the console fixed in the bottom around $4 \mathrm{~Hz}$, which is also close to the practical values. The top of the vertical beam is linked by rigid weightless beams to two nodes at the level 30 meters, modeling the supports 
of the portal beams, located on the external side of the RB containment shell. These supports are 30 meters far from the $\mathrm{RB}$ vertical beam along $O x$ axis.

Two horizontal beams of the portal are 20-meter long and go in parallel along $O x 10$ meters one from another. They are modeled by weightless flexible beams with circular crosssections (radius $1 \mathrm{~m}$ ). Two lumped masses 100 tones each (modeling the carriage) are placed in the far ends of the horizontal beams (looking from RB). This position may be changed, of course. These two masses have rotational inertia $300 \mathrm{t} \mathrm{m}^{2}$ around all axes. Each horizontal beam has two supports: one in RB (see above), another one on the special portal frame described below. Each of supports has sixcomponent spring enabling to model different conditions. Translational stiffness is great everywhere $(1 \mathrm{E} 13 \mathrm{kN} / \mathrm{m})$. Great rotational stiffness around $O x$ in the RB supports prevents from the rigid torsion of the beam. Other components of the rotational stiffness (two in $\mathrm{RB}$ support and three in the portal supports) are taken small (though nonzero, $10 \mathrm{kN} \mathrm{m} / \mathrm{rad})$. All beams in the ABAQUS model are threenode Timoshenko beams B32 [5]. All flexible beams have Young module $0.3 \mathrm{E} 8 \mathrm{kN} / \mathrm{m}^{2}$ and Poisson's coefficient 0.2 .

In practice there are usually two levels of the railways (one under another), but for the demonstration purposes one pair will also do.

Portal itself is modeled by the rectangular frame composed of vertical inertial beams 30-meter high with circular cross-section (radius 2 meters) in the $O y z$ plane. The frame is 10-meter wide-this is the distance between horizontal beams mentioned before. The mass density of the beams is $2.5 \mathrm{t} / \mathrm{m}^{3}$. The lower horizontal beam is weightless and rigid. The middle node of this beam is attached to the portal basement. Here the lumped mass 1650 tones is placed modeling the basement weight. This mass has the rotational inertia $300 \mathrm{t} \mathrm{m}^{2}$ around all axes.

Let us follow the algorithm of the dynamic stiffness condensation [2] to get structural matrix D. Using ABAQUS [5] two different modal analyses are performed. The first analysis is for the system with both basements (RB and TP) fixed. The first natural frequency is $2.4132 \mathrm{~Hz}$. In the corresponding natural mode only portal is deformed; masses on the top of portal have maximal displacements along $O y$. Next two natural modes show the deformation of the main building (i.e., RB).

The second ABAQUS modal analysis is performed for the system with only single base fixed (i.e., the RB basement). The problem here is that for the ideal hinges such system is a mechanism. If all the beams are rigid, such mechanism has four DOFs: portal frame has three DOFs (two translational and one rotational) in $O y z$ plane and one more rotational DOF around the upper beam of the frame. It means that four natural frequencies of the single-based system are zero. In our case (when hinges are not ideal) the first four natural frequencies are nonzero, though extremely low. As these frequencies are close to each other, the corresponding natural modes printed by ABAQUS are in fact the mixtures of the four "rigid" modes listed above.

In Table 1 one can see the ABAQUS results on natural frequencies for the two variants of the model fixing. For the comparison, the single RB natural frequencies (without portal) are also included.

We can conclude that the addition of portal (whether fixed or not) to the reactor building practically does not change the natural frequencies of RB. This is physically obvious taking into account huge difference in masses between $\mathrm{RB}$ and TP.

Inertial parameters of the "rigid" system ( similar for two fixing variants), natural frequencies with corresponding modal participation factors (different for two fixing variants), and modal displacements of the second support (in the single-support variant where the second support is not fixed) are read from the standard output ABAQUS files. Then they are treated according to [2] to get structural dynamic stiffness matrix $D$ in the frequency domain.

Now let us move to the dynamic stiffness matrix $S$ of the soil foundation. In the sample system let us consider both basements to rest on the surface of the soil. Base mat for the reactor building $(\mathrm{RB})$ is rectangular $60 \mathrm{~m} * 80 \mathrm{~m}$ (along $O x$ and $\mathrm{O} y$, resp.). Finite element (FE) mesh for it is $12 * 16$ elements (each element is $5 \mathrm{~m} * 5 \mathrm{~m}$ ). Base mat of the transportation portal (TP) is also rectangular $5 \mathrm{~m} * 30 \mathrm{~m}$ (see Figure 1). FE mesh for it consists of 6 elements of the same size $5 \mathrm{~m} * 5 \mathrm{~m}$. The distance between two base mat centers is $50 \mathrm{~m}$. Thus, we have $13 * 17+2 * 7=235$ "interaction" nodes on the surface of the soil foundation, common for soil and structure.

Let us consider the soil foundation composed of the $20 \mathrm{~m}$ thick layer resting on a homogeneous half-space. Mass density in the layer is $2 \mathrm{t} / \mathrm{m}^{3}$, shear wave velocity $V_{s}=$ $500 \mathrm{~m} / \mathrm{s}$, primary wave velocity $V_{p}=1100 \mathrm{~m} / \mathrm{s}$, and material damping is 0.07 . In the half-space mass density of the soil is $2.2 \mathrm{t} / \mathrm{m}^{3}$, shear wave velocity $V_{s}=900 \mathrm{~m} / \mathrm{s}$, primary wave velocity $V_{p}=2100 \mathrm{~m} / \mathrm{s}$, and material damping is 0.04 .

The approach to the soil condensed dynamic stiffness matrix evaluation is as follows [3]. The computer code SASSI [4] provides the full nodal complex impedance matrix in the frequency domain. It has size $705 * 705$ (each of 235 "interaction" nodes has 3 translational DOFs, total 705 DOFs). This matrix is calculated by standard SASSI. The author has added to SASSI the special option to condense this full matrix to one or several rigid basements (two basements - in our sample case). The condensed matrix has size $(6 N) *(6 N)$, where $N$ is the number of rigid basements. In our case the size is $12 * 12$, which corresponds to the size of the condensed matrix $D$ for the structure.

Figure 2 shows the translational dynamic stiffness along axes $\mathrm{Ox}, \mathrm{O} y, \mathrm{Oz}$ for the main structure $(\mathrm{RB})$ in the frequency domain. These are the first three diagonal elements of the submatrix $S^{11}$ in the soil matrix $S$. Here one can see also the corresponding elements of the structural dynamic stiffness submatrix $D^{11}$.

Figure 3 shows the translational dynamic stiffness along axes $\mathrm{Ox}, \mathrm{O} y, \mathrm{Oz}$ for the portal basement. These are the first three diagonal elements of the block $S^{22}$ in the soil stiffness matrix. In the whole matrix $S$ these are the seventh, the eighth, and the ninth diagonal elements (see the plot). One can compare them to the corresponding elements from 
TABLE 1: The comparison of the natural frequencies, Hz.

\begin{tabular}{|c|c|c|c|c|c|c|}
\hline \multicolumn{2}{|c|}{ RB standing alone } & \multicolumn{2}{|c|}{$\mathrm{RB}+\mathrm{TP}$, two basements fixed } & \multicolumn{2}{|c|}{$\mathrm{RB}+\mathrm{TP}$, one basement fixed } & \multirow[t]{2}{*}{ Comments about modes } \\
\hline No. & Frequency & No. & Frequency & No. & Frequency & \\
\hline & & & & 1 & $3.16 E-4$ & "Rigid" motion of portal \\
\hline & & & & 2 & $4.48 E-4$ & "Rigid" motion of portal \\
\hline & & & & 3 & $8.78 E-4$ & "Rigid" motion of portal \\
\hline & & & & 4 & $1.67 E-3$ & "Rigid" motion of portal \\
\hline & & 1 & 2.4132 & & & Lumped masses on the top of portal move along $O y$ \\
\hline 1 & 4.0445 & 2 & 4.0445 & 5 & 4.0445 & $\mathrm{RB}$ moves in the $O z y$ plane \\
\hline 2 & 4.0747 & 3 & 4.0647 & 6 & 4.0593 & $\mathrm{RB}$ moves in the $O z x$ plane \\
\hline 3 & 9.2132 & 6 & 9.2057 & 12 & 9.2137 & Torsion of RB around $\mathrm{Oz}$ \\
\hline 4 & 10.093 & 7 & 10.092 & 14 & 10.092 & Vertical movement of RB \\
\hline
\end{tabular}

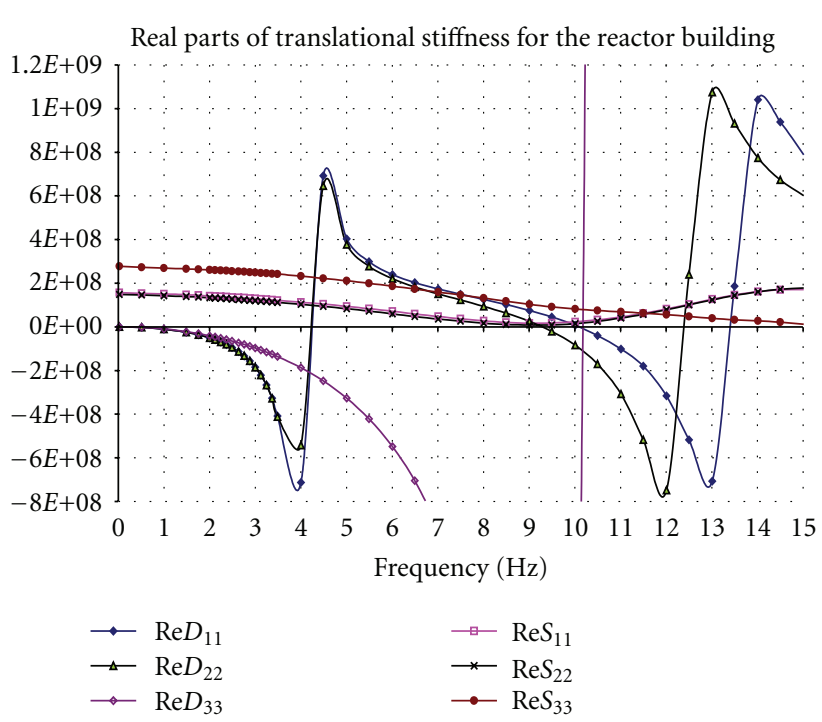

(a)

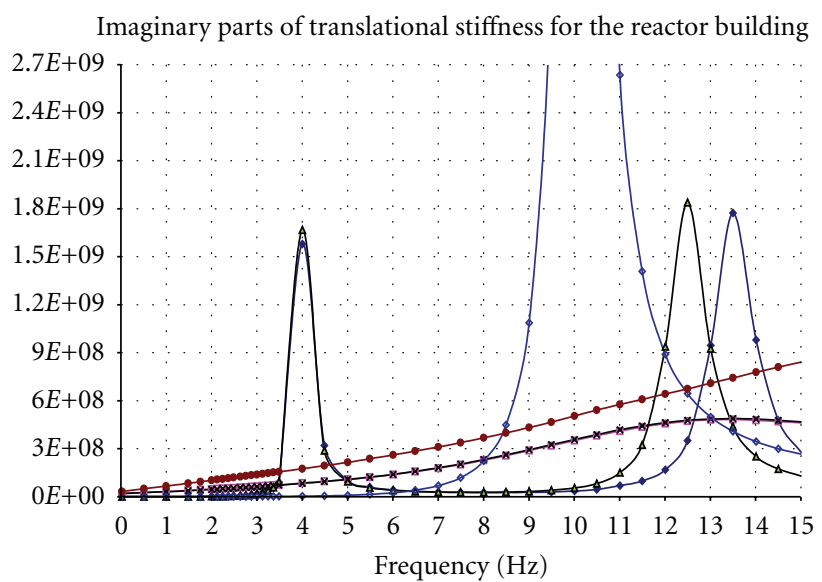

$\rightarrow \operatorname{Im} D_{11}$
$\rightarrow \operatorname{Im} D_{22}$
$\rightarrow \operatorname{Im} D_{33}$

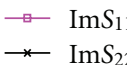$$
\rightarrow \operatorname{Im} S_{33}
$$

(b)

Figure 2: Translational dynamic stiffness of the soil and the structure along axes $O x, O y, O z$ for the reactor building basement.

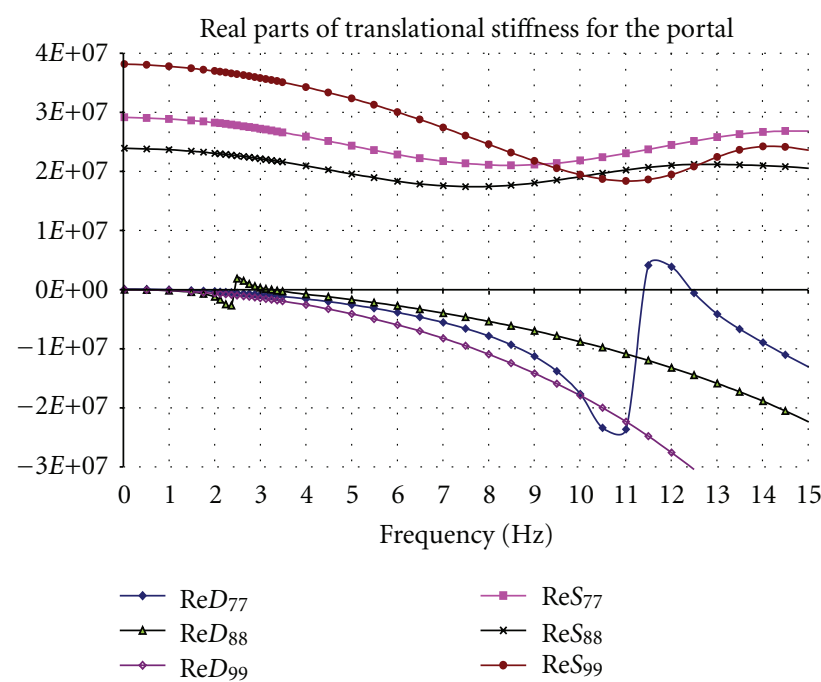

(a)

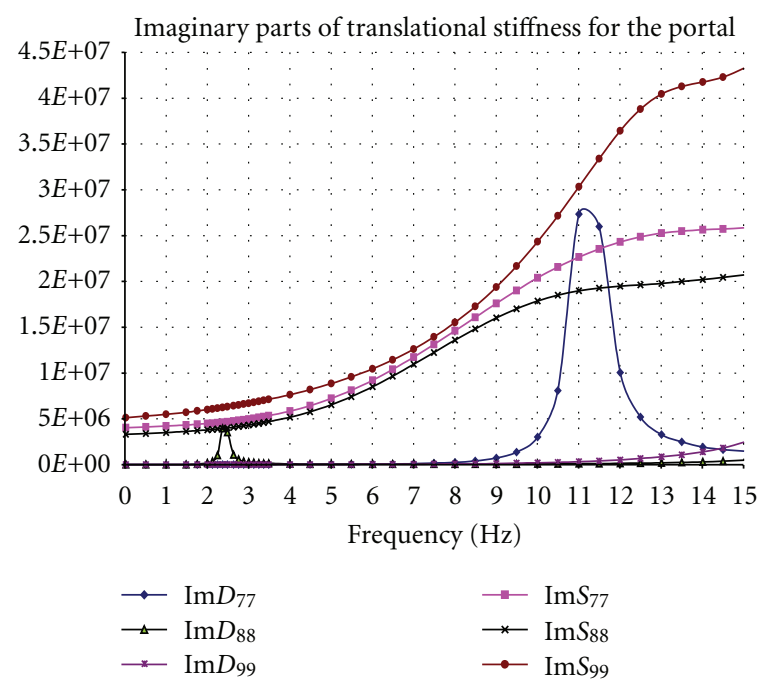

(b)

Figure 3: Translational dynamic stiffness of the soil and the structure along axes $O x, O y, O z$ for the portal basement. 


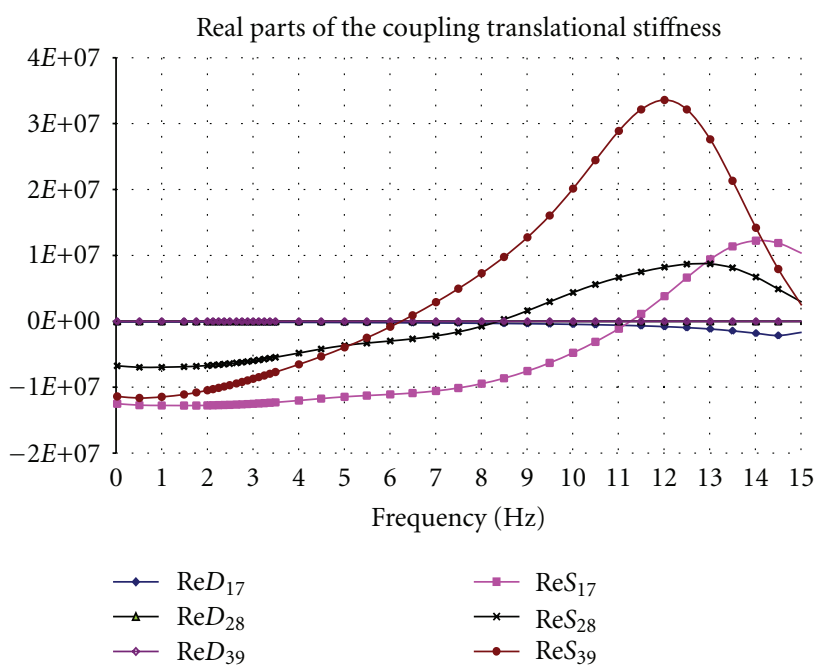

(a)

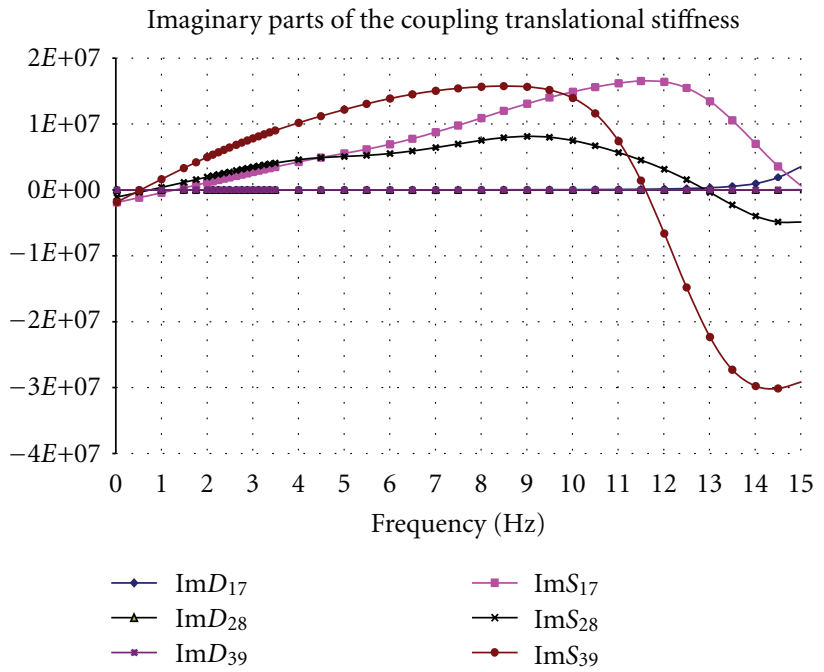

(b)

Figure 4: Coupling translational dynamic stiffness of the soil and the structure along axes $O x, O y, O z$.

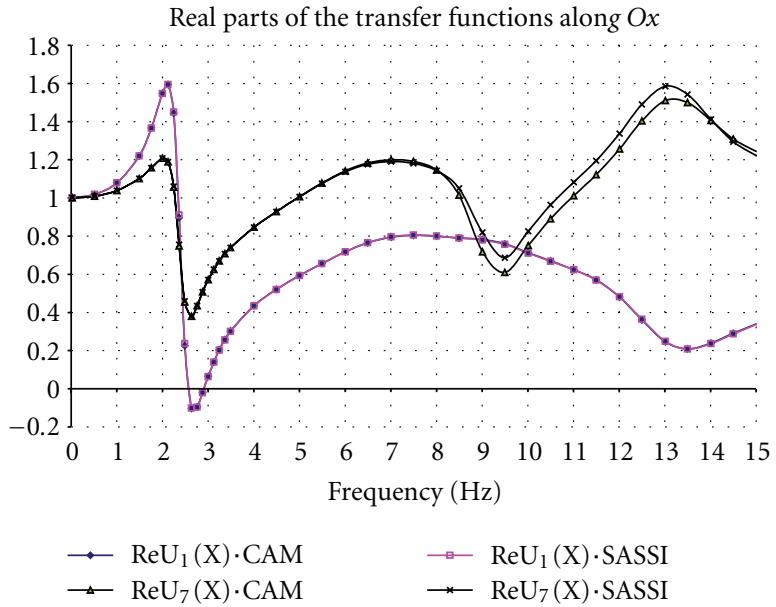

(a)

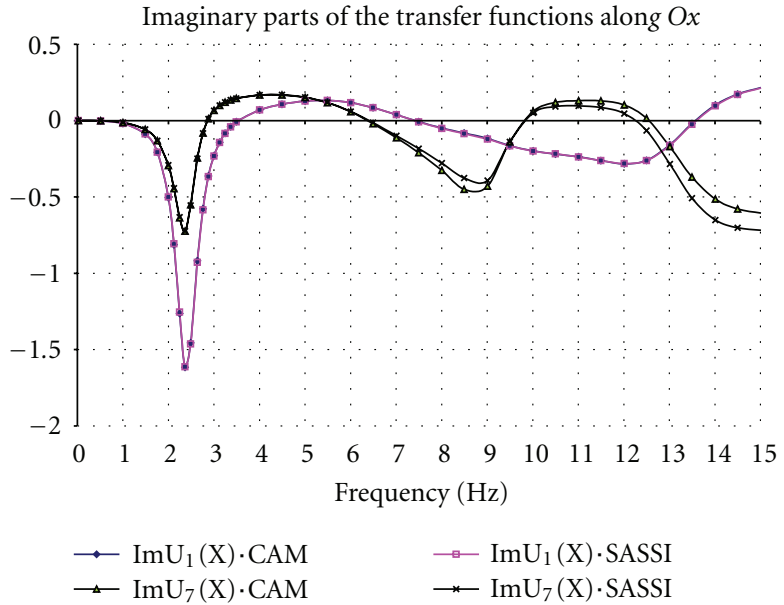

(b)

Figure 5: Transfer functions the free-field motion to the motion of the basements along $O x$.

the structural matrix $D$, preliminary shifted to the common coordinate system with $S$ (centered at the RB basement).

The difference between Figures 2 and 3 is not only in the scale (see vertical axes in the plots). We see that the real part of the total stiffness $(S+D)$ for RB will cross zero several times. For TP the real part of the soil stiffness $S$ (with fixed neighboring RB basement) is higher than that of the structural stiffness $D$, and the real part of the total stiffness $(S+D)$ will not cross zero in the frequency range below $9 \mathrm{~Hz}$. Roughly speaking, it means that for the low frequencies all resonances of the portal basement will only reflect the resonances of the reactor building.

Figure 4 shows the "coupling" translational dynamic stiffness along axes $O x, O y, O z$, reflecting the interaction of the two basements via the soil foundation. These are the first three diagonal terms of the block $S^{12}$ of the soil matrix $S$ (in $S$ they have numbers 1,$7 ; 2,8 ; 3,9$ used in the plot).
We can conclude that for the structure (due to the hinges) the back influence of the portal to the main building is insignificant (see $D_{17}, D_{28}, D_{39}$ ). However, the back influence of the portal basement to the RB basement through the soil foundation is far greater (see $S_{17}, S_{28}, S_{39}$ ). Note that all stiffness elements are the forces acting to one of the basements when one basement is fixed, and another one has a unit displacement. So, even the considerable coupling element in the stiffness matrix does not mean considerable interaction force, if both basements are moving together. It is reasonable that the comparatively light portal is unable to change the motion of the heavy reactor building.

Figure 5 shows the comparison of the transfer functions from the free-field motion to the motion of RB and TP along $O x$. On one hand these transfer functions were obtained by CAM (i.e., from (1)), and on the other hand, by the direct SASSI analysis without CAM. 
For the reactor building we see the full coincidence; for the portal we see fine matching, but with some difference. The additional analysis has proven that the reason for this difference is in the different treatment of the distributed masses in SASSI [4] and ABAQUS [5]. We see this difference in the portal only, because the beams in RB and horizontal beams are weightless. If portal is also modeled by weightless beams and lumped masses the difference is gone.

The similar results in two other directions are not shown here, but the coincidence of the transfer functions along $O y$ and $\mathrm{Oz}$ is even better than along $\mathrm{Ox}$.

\section{Conclusions}

The main result of this paper is the coincidence of the transfer functions obtained using CAM with the transfer functions obtained by the direct SASSI analysis. This result proves the applicability of the proposed method (extended CAM) at least for the double-based structures.

For the practical use the presented method still seems too sophisticated. However, the possible additional assumptions (e.g., about the master-slave relations between RB and portal, as well as about the absence of the structure-soil-structure interaction) may be justified against the full solution presented in this paper. The author plans to discuss these justifications in the future.

\section{References}

[1] A. G. Tyapin, "Combined asymptotic method for soil-structure interaction analysis," Journal of Disaster Research, vol. 5, no. 4, pp. 340-350, 2010.

[2] A. Tyapin, "Modal approach to the condensed dynamic stiffness evaluation," Journal of Mechanics Engineering and Automation, vol. 1, no. 1, 2011.

[3] A. Tyapin, "The frequency-dependent elements in the code SASSI: a bridge between civil engineers and the soil-structure interaction specialists," Nuclear Engineering and Design, vol. 237, no. 12-13, pp. 1300-1306, 2007.

[4] J. Lysmer et al., "SASSI-a computer system for dynamic soilstructure interaction analysis," Tech. Rep. UCB IGT/81-02, University of California, Berkeley, Calif, USA, 1981.

[5] ABAQUS, "Version 6.8," Dassault Systèmes Simulia Corp., Providence, RI, USA, 2008. 

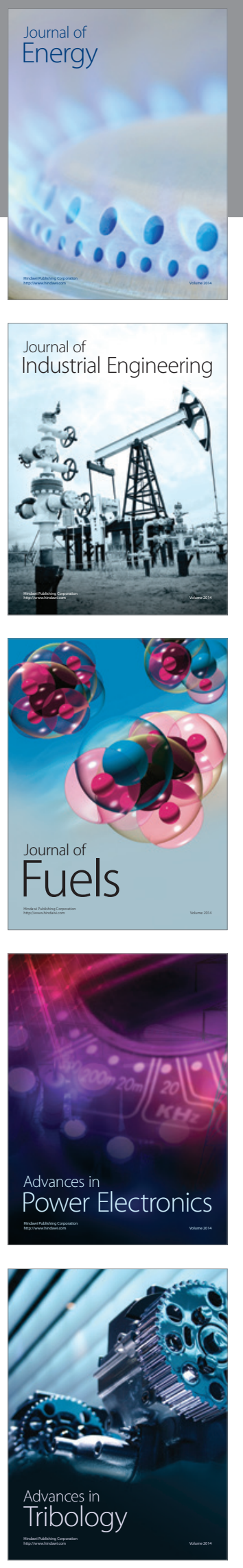
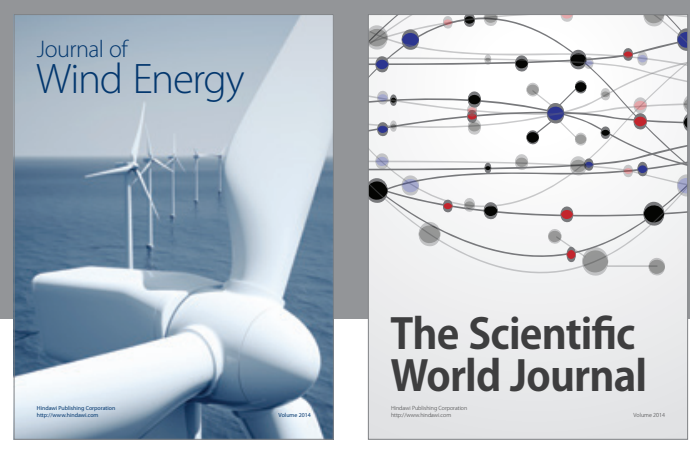

The Scientific World Journal

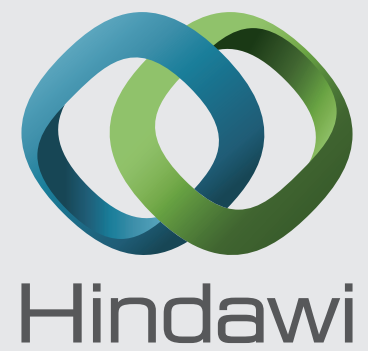

Submit your manuscripts at http://www.hindawi.com
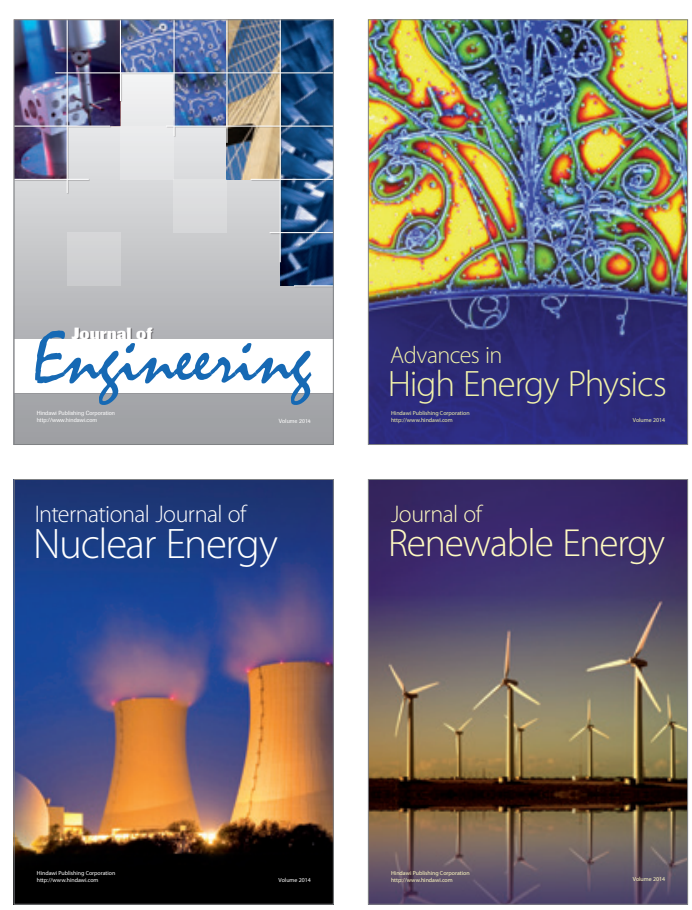

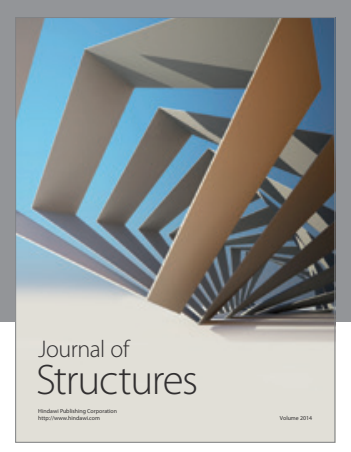

Rotating
Mechinery
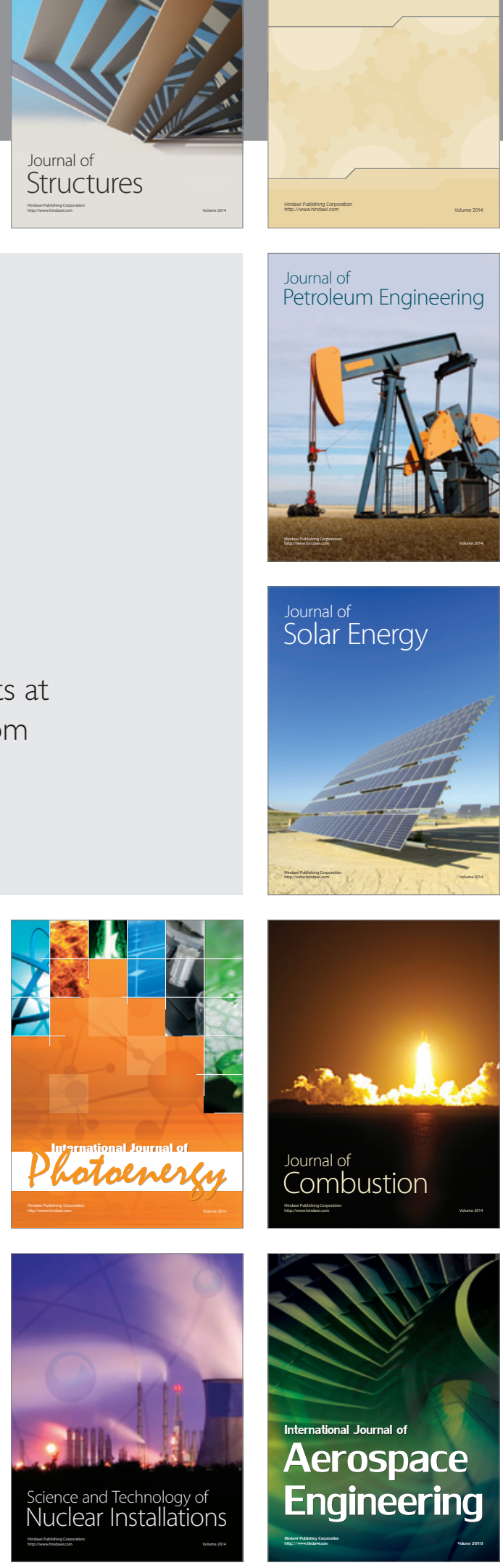